\title{
Treatment results including more than third-line chemotherapy for patients with advanced non-small cell lung cancer
}

\author{
SEH JONG PARK, IN KEUN CHOI, HEE YUN SEO, HWA JUNG SUNG, KYONG HWA PARK, \\ SANG CHEUL OH, JAE HONG SEO, SANG WON SHIN, YEUL HONG KIM and JUN SUK KIM
}

\author{
Department of Internal Medicine, Division of Hematology/Oncology, \\ College of Medicine, Korea University, Seoul, Korea
}

Received June 3, 2009; Accepted September 21, 2009

DOI: 10.3892/ol_00000009

\begin{abstract}
Recently, third-line chemotherapy for advanced nonsmall cell lung cancer (NSCLC) was accepted as a reasonable therapeutic option in patients with a favorable performance status. In practice, however, palliative chemotherapy has been performed for patients with a favorable performance status, even after third-line chemotherapy. Although multiple cycles of palliative chemotherapy were performed for these patients, there are little data of observation for courses of treatment from first-line to the last chemotherapy. We reviewed the courses of treatment for 82 patients with advanced NSCLC that had been admitted for platinum-based chemotherapy as a first-line treatment. Additional cycles of palliative chemotherapy were provided as monotherapy, based on the attending physician's decision considering patient performance status and toxicity after disease progression for previous chemotherapy. The median number of chemotherapy lines and cycles were 2 and 7, respectively, from first-line to the last chemotherapy. The median overall survival was 24 months in the response group of first-line chemotherapy, compared to 15 months for the entire study group. In the response group, the median number of chemotherapy cycles was 15 and patients received a median of 3 lines of chemotherapy. A total of 33 patients were candidate third-line chemotherapy or more. The median survival was 23 months for patients treated with more than third-line chemotherapy, compared to 7 months for patients treated with less than second-line chemotherapy. We conclude that long-standing chemotherapy is not beneficial to all NSCLC patients. However, patients with a favorable response to first-line chemotherapy tend to receive a higher number and more cycles of chemotherapy than the non-response group. Furthermore, multi-line chemotherapy appears to increase
\end{abstract}

Correspondence to: Dr In Keun Choi, Division of Medical Oncology, Korea University, Ansan Hospital, 516 Kojan-dong, Danwon-ku, Ansan-si, Kyoungki-do 425-707, Korea

E-mail:ink705@korea.ac.kr

Key words: more than third-line, chemotherapy, advanced, non-small cell lung cancer survival in the response group. Further studies will be needed to confirm these results.

\section{Introduction}

The role of chemotherapy in advanced lung cancer was not proven until the mid-1990s. In 1995, a meta-analysis by the Non-small Cell Lung Cancer Collaborative Group showed that cisplatin-based chemotherapy was effective in patients with advanced non-small cell lung cancer (NSCLC) (1). More recently, in 2000, second-line chemotherapy with docetaxel was shown to prolong survival in NSCLC patients previously treated with platinum-based chemotherapy as a first-line chemotherapy treatment (2). As more novel agents have been been shown to be effective against lung cancer, such as EGFR inhibitors, third-line chemotherapy can be accepted as a reasonable therapeutic option $(3,4)$. These types of chemotherapy were also shown to improve quality of life (QOL) (5).

The role of additional palliative chemotherapy for patients with progressive disease after third-line chemotherapy remains unclear. If additional chemotherapy improves survival or QOL of patients even after third-line chemotherapy, further treatment may be a reasonable option in patients with a favorable performance status. However, little data are available on the results of additional treatments including more than third-line chemotherapy. Therefore, we report on treatment outcomes including more than third-line chemotherapy for advanced NSCLC patients.

\section{Patients and methods}

Eighty-two patients with inoperable advanced NSCLC were admitted for platinum-based chemotherapy as a first-line treatment at the Korea University Hospitals between March 2003 and February 2007. All 82 patients were initially treated with cisplatin-based first-line chemotherapy in combination with gemcitabine or taxanes. When disease progression was confirmed, the patients moved on to additional lines of chemotherapy with drugs such as docetaxel, gefitinib or erlotinib, vinorelbine, irinotecan and pemetrexed. Vinorelbine and irinotecan were known to be effective in combination with the platinum agent in first-line chemotherapy (6). These drugs were provided as monotherapy based on the attending 
physician's decision based on patient performance status and drug toxicity. Selection of drugs with same toxicity profiles as the previous treatment was avoided to minimize cumulative toxicity. After every 2 cycles of chemotherapy, the patients were evaluated for a response. Toxicity was evaluated after every cycle of chemotherapy. The total cycles of chemotherapy and withdrawal of treatment were based on the physician's decision, as well as the benefit of treatment and risk of toxicity. Chemotherapy was changed or withdrawn if disease progression or unacceptable toxicity was observed. In cases of grade 4 hematological toxicities and more than a grade 3 nonhematological toxicity, the chemotherapy dose was reduced by $25 \%$.

The electronic medical records of the enrolled patients were retrospectively reviewed using the hospital's computer network. Information on the patients including performance status, based on the Eastern Cooperative Oncology Group (ECOG); performance scale; histological type of malignancy, chemotherapy regimens; overall survival; initial response; duration of response; total chemotherapy treatment lines and number of cycles of treatment were recorded. Collected information was then analyzed and evaluated for overall survival, duration of response and the total number of chemotherapy cycles provided. Patients with an initial response of partial response or better were further classified as the response group. The subgroup analysis for overall survival, total number of chemotherapy cycles and duration of initial response was determined and compared among the different response groups. In addition, a subgroup analysis for overall survival between smokers and non-smokers was performed.

The response to chemotherapy was classified according to the Response Evaluation Criteria in Solid Tumors criteria (7). Definitions of complete response (CR), partial response (PR), stable disease (SD) and progressive disease (PD) were as follows: CR, disappearance of all target lesions; PR, at least a $30 \%$ decrease in the sum of the longest diameters of target lesions compared to the baseline; PD, at least a $20 \%$ increase in the sum of the longest diameters of the target lesions, using as a reference the smallest sum of the longest diameters recorded since onset of the treatment or the appearance of one or more new lesions and SD, neither sufficient shrinkage to qualify for PR nor sufficient increase to qualify for PD.

The survival curves were plotted according to the method of Kaplan-Meyer. Log-rank and Chi-square analyses were performed to determine statistical significance.

\section{Results}

The median age of the enrolled patients was 65 and there were more males. At the time of diagnosis most patients had stage IV lung cancer except for three patients with stage IIIb disease. Most patients had a favorable performance status. Adenocarcinoma (47.5\%) was the most common histological type of cancer, followed by squamous cell carcinoma (26.8\%; Table I).

All patients started first-line chemotherapy with a regimen of cisplatin combined with either gemcitabine $(56.1 \%)$ or taxanes $(43.9 \%)$. Thirty-three out of 82 patients $(40.2 \%)$ had a PR or better on their initial response evaluation, including two patients who were observed with a CR. The median number of chemo-
Table I. Patient characteristics.

\begin{tabular}{|c|c|}
\hline No. of patients & 82 \\
\hline Median age & $65(23-86)$ \\
\hline \multicolumn{2}{|l|}{ Gender } \\
\hline Male & $24(29.3 \%)$ \\
\hline Female & $58(70.1 \%)$ \\
\hline \multicolumn{2}{|l|}{ Stage } \\
\hline $\mathrm{IIIb}$ & $3(3.7 \%)$ \\
\hline IV & $79(96.3 \%)$ \\
\hline \multicolumn{2}{|l|}{ Performance status (ECOG) } \\
\hline 0 & $14(17.1 \%)$ \\
\hline 1 & $40(48.8 \%)$ \\
\hline 2 & $21(25.6 \%)$ \\
\hline 3 & $7(8.5 \%)$ \\
\hline \multicolumn{2}{|l|}{ Histological type of NSCLC } \\
\hline Adenocarcinoma & $39(47.5 \%)$ \\
\hline Squamous cell carcinoma & $22(26.8 \%)$ \\
\hline Poorly differentiated & $19(23.2 \%)$ \\
\hline Large cell carcinoma & $2(2.4 \%)$ \\
\hline \multicolumn{2}{|l|}{ First-line chemotherapy regimen } \\
\hline Gemcitabine + cisplatin & $46(56.1 \%)$ \\
\hline Taxene + cisplatin & $36(43.9 \%)$ \\
\hline \multicolumn{2}{|c|}{ Initial response of first-line chemotherapy } \\
\hline $\mathrm{CR}$ & $2(2.4 \%)$ \\
\hline PR & $31(37.8 \%)$ \\
\hline SD & $26(31.7 \%)$ \\
\hline PD & $23(28.0 \%)$ \\
\hline Median no. of chemotherapy lines & $2(1-8)$ \\
\hline Median no. of chemotherapy cycles & $7(1-33)$ \\
\hline Median overall survival & 15 months \\
\hline $\begin{array}{l}\text { No. of patients treated with } \\
\text { third-line chemotherapy or more }\end{array}$ & $33(40.2 \%)$ \\
\hline
\end{tabular}

$\mathrm{CR}$, complete response; PR, partial response; $\mathrm{SD}$, stable disease; $\mathrm{PD}$, progressive disease.

therapy lines and cycles received were 2 and 7, respectively. A total of 33 patients were candidate third-line chemotherapy or more. The highest number of lines and cycles of chemotherapy was eighth-line and total 33 cycles (Table I). The median overall survival of the studied patients was 15 months (Fig. 1A).

Overall survival according to response in the first line chemotherapy. In the subgroup analysis, a total of 33 patients were classified as the response group for first-line chemotherapy. The median overall survival was increased to 24 months $(95 \%$ CI, 18.8-29.2) in the response group compared to 15 months (95\% CI, 4.7-25.3) for the entire study group. Fig. 1 shows that survival increased as the response to initial chemotherapy improved. The patients of the response groups in first-line chemotherapy received on average 1 more additional line of chemotherapy compared to the entire group of patients. This group received a median of 3 lines of chemotherapy. The median number of chemotherapy cycles received was 15 in the 


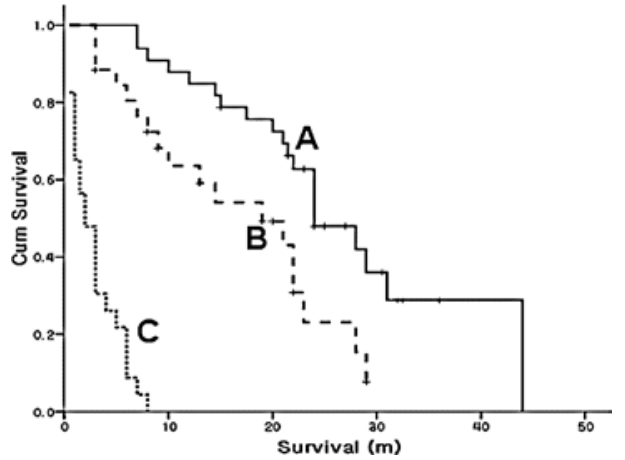

Figure 1. Survival curves according to the initial response to the initial chemotherapy. (A) The median overall survival was increased to 24 months (95\% CI, 18.8-29.2) in the response group of the initial first-line chemotherapy. (B) The median overall survival was 19 months (95\% CI, 8.7-29.3) in the stable disease group and (C) only 2 months (95\% CI, 0.8-3.2) for the progressive disease group.

Table II. Median survival, duration of initial response, median number of chemotherapy lines and cycles in all patients and the response group.

\begin{tabular}{lcc}
\hline & All patients & Response \\
\hline No. of patients & 82 & 33 \\
Median overall survival (months) & 15 & 24 \\
Median no. of chemotherapy lines & 2 & 3 \\
Median no. of chemotherapy cycles & 7 & 15 \\
\hline
\end{tabular}

response group. The median number of chemotherapy cycles was 7 for the 82 patients (Table II).

Number of chemotherapy lines. The subgroup analysis showed that 49 patients were treated with less than secondline chemotherapy and 33 patients $(40.2 \%)$ were treated with more than third-line chemotherapy. The median survival was 23 months (95\% CI, 20.9-25.1) in patients treated with more than third-line chemotherapy, compared to 7 months $(95 \% \mathrm{CI}$, 4.7-9.3) in patients treated with less than second-line chemotherapy (Fig. 2).

Smokers and non-smokers. Fifty-three smokers and 29 nonsmokers participated in this study. A significant difference of 10 and 28 months, respectively, was noted in the median overall survival of smokers compared to non-smokers (Fig. 3).

Toxicities. Concerns were raised regarding the increase of cumulative toxicities in patients treated with multiple lines and cycles of chemotherapy. Although nephrotoxicity, neurotoxicity and bone marrow suppression occurred during additional cycles of chemotherapy, no unmanageable cumulative toxicities among patients treated with additional chemotherapy were noted. Selection of drugs with same toxicity profiles as the previous treatment was avoided to minimize cumulative toxicity. Monotherapy may also contribute to decreasing the toxicity of additional chemotherapy (data not shown).

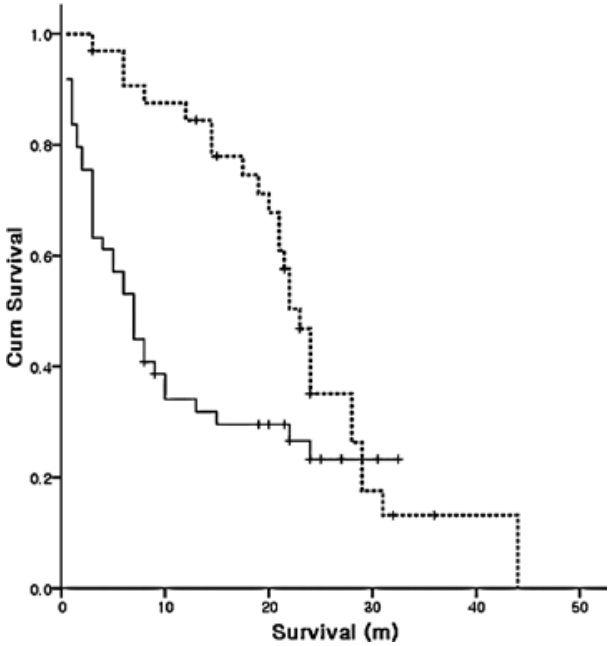

Figure 2. Survival curves according to the lines of chemotherapy. The median survival was 23 months (95\% CI, 20.9-25.1) in patients treated with more than third-line chemotherapy, compared to 7 months (95\% CI, 4.7-9.3) in patients treated with less than second-line chemotherapy.

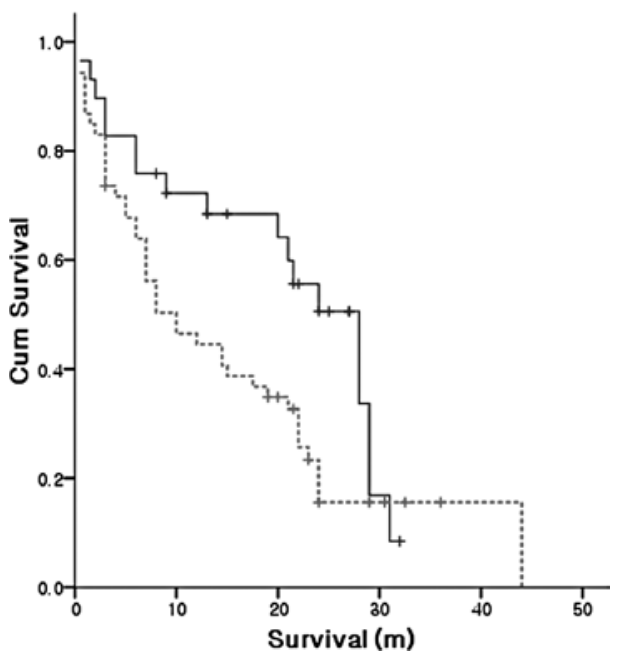

Figure 3. Survival curves according to the smoking status of the patients. A significant difference of 10 months (95\% CI, 4.2-15.2) and 28 months (95\% CI, 21.1-34.9), respectively, was noted in the median overall survival of smokers compared to non-smokers.

\section{Discussion}

In the treatment of patients with inoperable, advanced lung cancer, first- and second-line chemotherapy have proven to be effective $(1,2)$. Agents such as docetaxel, pemetrexed and EGFR inhibitors are widely used for second- and third-line chemotherapy $(3,4,8)$. However, even after completion of third-line chemotherapy, some patients still exhibit a favorable performance status despite disease progression. The question is whether present with a favorable performance status after third-line chemotherapy. Chemotherapy beyond third-line treatment is supported by medical insurance in Korea. Thus, in practice, when these treatments are shown to improve QOL even after third-line chemotherapy, a physician may approve additional cyles of chemotherapy after disease progression. 
In this study, the subgroup analysis showed that 49 patients were treated with less than second-line chemotherapy and 33 patients $(40.2 \%)$ were treated with more than third-line chemotherapy. This result is similar to that of Murillo and Koeller, who also reported that $41 \%$ patients received more than third-line chemotherapy (9).

Vigorous efforts are ongoing to prolong survival by increasing drug exposure or duration. Sequential, alternating and maintenance therapy have been proposed in an attempt to improve survival. These treatments are appealing as they may increase the number of non-cross resistant agents and/or duration of chemotherapy. As maintenance therapy, paclitaxel has shown success (10-12), whereas agents such as docetaxel and gemcitabine have failed to show significant benefits $(10,13,14)$. Based on the studies published thus far, however, it is unlikely that sequential and alternating treatment will prove to be superior to the current standard therapy for all advanced NSCLC patients (15). However, selective groups, especially chemo-sensitive patients, may benefit from such treatments.

In this retrospective study, the overall treatment results for patients with inoperable advanced NSCLC had a median overall survival of 15 months. This finding was not significantly different from the results of previous reports. However, in the subgroup analysis of patients with a partial response or better (response group) to first-line chemotherapy, the median overall survival increased to 24 months. Compared to the entire study group, patients of the response group in first-line chemotherapy received a median of 1 more line and 8 more cycles of chemotherapy. They were exposed to more chemotherapeutic agents than the non-responsive group. The patient, for example, who was treated with the highest number of chemotherapy lines and cycles in this study received as many as 8 lines and 33 cycles of chemotherapy. The median survival was 23 months in patients treated with more than third-line chemotherapy, compared to 7 months in patients treated with less than second-line chemotherapy.

Notably, since the patients lived longer, they could be potential candidates for additional chemotherapy. However, the increase of the median overall survival of 24 months in the response group is difficult to explain solely by additional chemotherapy. In a study that identified an objective response to chemotherapy as a marker for further survival, a median survival time of 41 and 19 weeks was reported for the responding and non-responding patients, respectively (16).

For patients with colorectal cancer, exposure to 3 active cytotoxic drugs prolonged survival regardless of whether the drugs were administered as monotherapy or in combination (17-20, Pitot HC, et al, J Clin Oncol 23: abs. 3506, 2005). It is possible that a similar mechanism applies to patients with NSCLC. Multi-drug exposure shown to be non-cross resistant may increase survival in the response group. If this is also true for NSCLC patients, additional chemotherapy after secondor third-line chemotherapy may be an effective and feasible strategy for treating patients with inoperable NSCLC. Careful patient selection is important, since many of the patients that were resistant to the initial chemotherapeutic agents appeared to be resistant to second- and third-line drugs as well.

The subgroup analysis comparing the survival of NSCLC patients who were smokers to non-smokers showed that the smokers had a significantly worse prognosis. This finding is comparable to the results of previously published studies (21). Certain investigators have suggested that NSCLC among nonsmokers exhibits different clinical and pathological features compared to that of smokers (22-24).

Treatment-related toxicities are always a concern when further chemotherapy is required. Although not studied intensively, we did not encounter unmanageable toxicities associated with increased chemotherapy exposure and related cumulative toxicities. The fact that most of the additional lines were single agent lines of chemotherapy may have played a role in reducing the toxicities. In addition, chemotherapeutic agents were carefully selected in order that the major toxicities of these drugs did not overlap the major toxicities of previously used drugs.

Significant limitations of this study were that it was not a prospective randomized study, and that the sample size is small. However, the results suggested that NSCLC patients with a favorable response to initial chemotherapy benefit from further chemotherapy beyond third-line treatment by multidrug exposure. Chemotherapy may, therefore, benefit NSCLC patients who remain fit for treatment following the completion of third-line cycles of chemotherapy. Prospective large scale studies are needed to confirm these results.

\section{References}

1. Non-small Cell Lung Cancer Collaborative Group: Chemotherapy in non-small cell lung cancer: a meta-analysis using updated data on individual patients from 52 randomised clinical trials. BMJ 311: 899-909, 1995.

2. Shepherd FA, Dancey J, Ramlau R, et al: Prospective randomized trial of docetaxel versus best supportive care in patients with nonsmall-cell lung cancer previously treated with platinum-based chemotherapy. J Clin Oncol 18: 2095-2103, 2000.

3. Cappuzzo F, Gregorc V, Rossi E, et al: Gefitinib in pretreated non-small cell lung cancer (NSCLC): analysis of efficacy and correlation with HER2 and epidermal growth factor receptor expression in locally advanced or metastatic NSCLC. J Clin Oncol 21: 2658-2663, 2003.

4. Shepherd FA, Rodrigues Pereira J, Ciuleanu T, et al: Erlotinib in previously treated non-small cell lung cancer. N Engl J Med 353: 123-132, 2005.

5. Bunn PA Jr and Kelly K: New chemotherapeutic agents prolong survival and improve quality of life in non-small cell lung cancer: a review of the literature and future directions. Clin Cancer Res 4: 1087-1100, 1998.

6. Ohe Y, Ohashi Y, Kubota K, et al: Randomized phase III study of cisplatin plus irinotecan versus carboplatin plus paclitaxel, cisplatin plus gemcitabine, and cisplatin plus vinorelbine for advanced non-small cell lung cancer: Four-Arm Cooperative Study in Japan. Ann Oncol 18: 317-323, 2007.

7. Therasse P, Arbuck SG, Eisenhauer EA, et al: New guidelines to evaluate the response to treatment in solid tumors. European Organization for Research and Treatment of Cancer, National Cancer Institute of the United States, National Cancer Institute of Canada. J Natl Cancer Inst 92: 205-216, 2000.

8. Hanna N, Shepherd FA, Fossella FV, et al: Randomized phase III trial of pemetrexed versus docetaxel in patients with non-small cell lung cancer previously treated with chemotherapy. J Clin Oncol 22: 1589-1597, 2004.

9. Murillo JR Jr and Koeller J: Chemotherapy given near the end of life by community oncologists for advanced non-small cell lung cancer. Oncologist 11: 1095-1099, 2006.

10. Belani CP, Barstis J, La Rocca RV, et al: Meta-analysis of weekly paclitaxel as maintenance therapy for advanced non-small cell lung cancer patients following initial chemotherapy. Lung Cancer 49: 33, 2005.

11. Belani CP, Barstis J, Perry MC, et al: Multicenter, randomized trial for stage IIIB or IV non-small cell lung cancer using weekly paclitaxel and carboplatin followed by maintenance weekly paclitaxel or observation. J Clin Oncol 21: 2933-2939, 2003. 
12. Ramalingam S and Belani C: Systemic chemotherapy for advanced non-small cell lung cancer: recent advances and future directions. Oncologist 13: 5-13, 2008.

13. Brodowicz T, Krzakowski M, Zwitter M, et al: Cisplatin and gemcitabine first-line chemotherapy followed by maintenance gemcitabine or best supportive care in advanced non-small cell lung cancer: a phase III trial. Lung Cancer 52: 155-163, 2006.

14. Fidias P, Dahkil SR, Lyss AP, et al: Updated report of a phase III study of induction therapy with gemcitabine + carboplatin (GC) followed by either delayed vs. immediate second-line therapy with docetaxel (D) in advanced non-small cell lung cancer. J Clin Oncol 24: 7032, 2006.

15. Grossi F, Aita M, Follador A, et al: Sequential, alternating and maintenance/consolidation chemotherapy in advanced non-smal cell lung cancer: a review of the literature. Oncologist 12: 451-464, 2007.

16. Paesmans M, Sculier JP, Libert P, et al: Response to chemotherapy has predictive value for further survival of patients with advanced non-small cell lung cancer: 10 years experience of the European Lung Cancer Working Party. Eur J Cancer 33: 2326-2332, 1997.

17. Goldberg RM, Sargent DJ, Morton RF, et al: A randomized controlled trial of fluorouracil plus leucovorin, irinotecan and oxaliplatin combinations in patients with previously untreated metastatic colorectal cancer. J Clin Oncol 22: 23-30, 2004.

18. Grothey A and Sargent D: Overall survival of patients with advanced colorectal cancer correlates with availability of fluorouracil, irinotecan and oxaliplatin regardless of whether doublet or single-agent therapy is used first line. J Clin Oncol 23: 9441-9442, 2005.
19. Grothey A, Sargent D, Goldberg RM and Schmoll HJ: Survival of patients with advanced colorectal cancer improves with the availability of fluorouracil-leucovorin, irinotecan and oxaliplatin in the course of treatment. J Clin Oncol 22: 1209-1214, 2004.

20. Tournigand C, Andre T, Achille E, et al: FOLFIRI followed by FOLFOX6 or the reverse sequence in advanced colorectal cancer: a randomized GERCOR study. J Clin Oncol 22: 229-237, 2004.

21. Scagliotti GV, Parikh P, Von Pawel J, et al: Phase III study comparing cisplatin plus gemcitabine with cisplatin plus pemetrexed in chemotherapy-naive patients with advanced-stage non-small cell lung cancer. J Clin Oncol 26: 3543-3551, 2008.

22. Einhorn LH: First-line chemotherapy for non-small cell lung cancer: is there a superior regimen based on histology? J Clin Oncol 26: 3485-3486, 2008.

23. Yano T, Miura N, Takenaka T, et al: Never-smoking non-small cell lung cancer as a separate entity: clinicopathologic features and survival. Cancer 113: 1012-1018, 2008.

24. Zhou W, Heist RS, Liu G, et al: Smoking cessation before diagnosis and survival in early stage non-small cell lung cancer patients. Lung Cancer 53: 375-380, 2006. 